\title{
Electron cyclotron resonance heating on TEXTOR*
}

\author{
E. Westerhof ${ }^{1}$, J.A. Hoekzema ${ }^{2}$, G.M.D. Hogeweij ${ }^{1}$, \\ R.J.E. Jaspers ${ }^{1}$, F.C. Schüller ${ }^{1}$, C.J. Barth ${ }^{1}$, W.A. Bongers ${ }^{1}$, \\ A.J.H. Donné ${ }^{1}$, P. Dumortier ${ }^{3}$, A.F. van der Grift ${ }^{1}$, \\ J.C. van Gorkom ${ }^{1}$, D. Kalupin ${ }^{3}$, H.R. Koslowski ${ }^{2}$, \\ A. Krämer-Flecken ${ }^{2}$, O.G. Kruijt ${ }^{1}$, N.J. Lopes Cardozo ${ }^{1}$, \\ P. Mantica ${ }^{4}$, H.J. van der Meiden ${ }^{1}$, A. Merkulov ${ }^{1}$, A. Messiaen ${ }^{3}$, \\ J.W. Oosterbeek ${ }^{2}$, T. Oyevaar ${ }^{1}$, A.J. Poelman ${ }^{1}$, R.W. Polman ${ }^{1}$, \\ P.R. Prins ${ }^{1}$, J. Scholten ${ }^{1}$, A.B. Sterk ${ }^{1}$, C.J. Tito ${ }^{1}$, V.S. Udintsev ${ }^{1}$, \\ B. Unterberg ${ }^{2}$, M. Vervier ${ }^{3}$, G. van Wassenhove ${ }^{3}$ and TEC Team \\ ${ }^{1}$ FOM-Institute for Plasma Physics Rijnhuizen, Association EURATOM-FOM, \\ PO Box 1207, 3430 BE Nieuwegein, The Netherlands (www.rijnh.nl) ${ }^{\mathrm{a}}$ \\ ${ }^{2}$ Institut für Plasmaphysik, Forschungszentrum Jülich, Euratom Association, D-52425 \\ Jülich, Germany ${ }^{\mathrm{a}}$ \\ ${ }^{3}$ Laboratoire de Physique des Plasmas-Laboratorium voor Plasmafysica, \\ Association Euratom-Belgian State, ERM/KMS, Brussels, Belgium ${ }^{\mathrm{a}}$ \\ ${ }^{4}$ Istituto di Fisica del Plasma, Ass. Euratom-ENEA-CNR, Milano, Italy
}

Received 6 December 2002, accepted for publication 12 September 2003

Published 3 November 2003

Online at stacks.iop.org/NF/43/1371

\begin{abstract}
The $110 \mathrm{GHz}$ and the new $140 \mathrm{GHz}$ gyrotron systems for electron cyclotron resonance heating (ECRH) and ECCD on TEXTOR are described and results of ECRH experiments with the $110 \mathrm{GHz}$ system are reported. Central ECRH on Ohmic plasmas shows the presence of an internal electron transport barrier near $q=1$. This is confirmed by modulated ECRH experiments. A central barrier is also indicated by ECRH in radiatively improved (RI) mode discharges and up to two barriers are seen with ECRH during the current ramp phase. ECRH control of sawteeth is reported for both Ohmic and RI mode target plasmas.
\end{abstract}

PACS numbers: $52.5 . \mathrm{Sw}$, 52.55.Fa, 52.25.Fi

\section{Introduction}

After the closure of the Rijnhuizen tokamak project (RTP) in 1998, the high temperature plasma physics research of the FOM-Instituut voor Plasmafysica 'Rijnhuizen', which is focused on detailed electron transport, has been concentrated on the TEXTOR tokamak at the Forschungszentrum Jülich, in the framework of the Trilateral Euregio Cluster (TEC) agreement. One of the major contributions of the FOM team is a $>800 \mathrm{~kW}, 140 \mathrm{GHz}, 10 \mathrm{~s}$, electron cyclotron resonance heating (ECRH) system, that is being installed during the shutdown for installation of the dynamic ergodic divertor

\footnotetext{
* This paper is an expanded version of the two papers presented on the TEXTOR ECRH system (J.A. Hoekzema et al ) and experimental results (E. Westerhof et al) at the 12th Joint Workshop on Electron Cyclotron Emission and Electron Cyclotron Resonance Heating (Aix-en-Provence, France, 13-16 May 2002).

a Partners in the Trilateral Euregio Cluster.
}

(DED) [1]. The divertor coils are installed on the high field side inside the vessel. In preparation of experiments with the new ECRH system, the $500 \mathrm{~kW}, 110 \mathrm{GHz}, 200 \mathrm{~ms}$ gyrotron, which had been in operation on the RTP tokamak since 1994, was installed on TEXTOR. In addition, a number of high resolution electron diagnostics have been installed on TEXTOR. In particular, these include a TV Thomson scattering (TVTS) system with a radial resolution of $8 \mathrm{~mm}$ [2].

TEXTOR is a limiter tokamak with $R_{0}=1.75 \mathrm{~m}, a=$ $0.46 \mathrm{~m}, I_{\mathrm{p}}<0.8 \mathrm{MA}, B_{0}<2.8 \mathrm{~T}$ and a pulse length $<10$ s. It already had additional heating with neutral beam injection $(\mathrm{NBI})(2 \times 1.5 \mathrm{MW})$, both beams being injected tangentially, one in the direction of the plasma current, the other in the opposite direction, and ion cyclotron resonance heating $(\mathrm{ICRH})(2 \times 2 \mathrm{MW})$. The usual toroidal field for operation is around $2.3 \mathrm{~T}$. The $110 \mathrm{GHz}$ frequency of the preliminary ECRH system is therefore suitable for second 


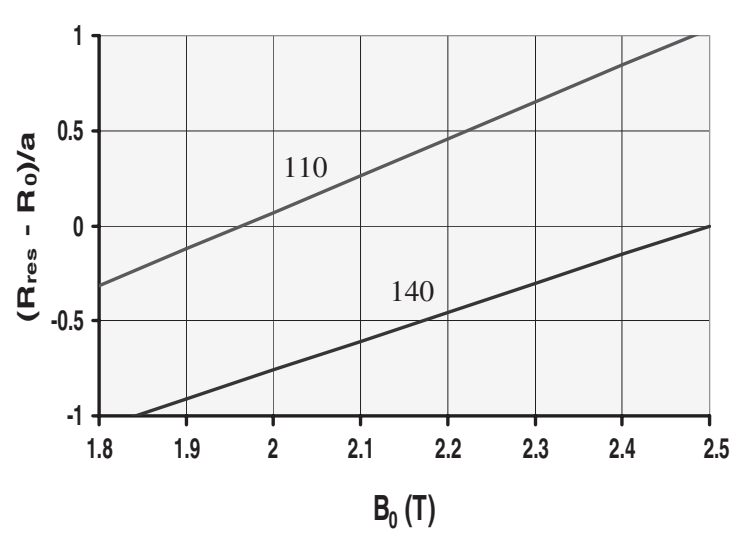

Figure 1. Resonance position for the $110 \mathrm{GHz}$ as well as the $140 \mathrm{GHz}$ waves as a function of toroidal magnetic field for perpendicular injection.

harmonic heating at reduced field $(\sim 2 \mathrm{~T}$, see figure 1$)$. Access to the enhanced confinement mode of TEXTOR, the radiatively improved (RI) mode [3] is limited due to the second harmonic $\mathrm{X}$-mode density limit. In the RI mode, densities above the Greenwald density limit are reached. For the new system, a frequency of $140 \mathrm{GHz}$ was selected to enable second harmonic (X-mode) central heating and especially current drive at the usual value of the toroidal field. For this frequency, the plasma is accessible up to the highest (RI mode) densities. At slightly reduced field, the interaction of ECRH with magnetic islands and ergodic field regions near the inner wall, as created and controlled by the DED, can be studied. For the $110 \mathrm{GHz}$ system, it is also possible to remove resonances from the plasma, and obtain a minimum of (ECE) background radiation, by increasing the field to $2.6 \mathrm{~T}$. This makes it possible to use the gyrotron as a source for collective Thomson scattering (CTS). Results of a first series of successful CTS experiments are reported elsewhere [4]. After installation of the new $140 \mathrm{GHz}$ gyrotron, the $110 \mathrm{GHz}$ gyrotron will remain operational for CTS campaigns, for which a collaboration with Ris $\varnothing$ and MIT exists. Because the gyrotrons share some of the auxiliary supplies, it will not be possible to operate the two gyrotrons simultaneously.

The first part of the paper (section 2) presents the technical set-up of the ECW systems: both of the preliminary $110 \mathrm{GHz}$ gyrotron as well as the new $140 \mathrm{GHz}$ gyrotron. The emphasis throughout this section is on the new $140 \mathrm{GHz}$ gyrotron. In particular, the following topics are dealt with: the gyrotrons and their auxiliary supplies including high voltage and cooling; the transmission line, which is largely shared between the two systems; the launcher, including the necessary modifications to make the launcher compatible with the high power, long pulses of the new gyrotron; and finally the control and data acquisition system. In the second part, section 3, the experimental results that have been obtained with the preliminary $110 \mathrm{GHz}$ system are described: the presence of transport barriers is probed by scanning of the ECRH deposition region in Ohmic plasmas. This clearly reveals the barrier at $q=1$, which has also been observed on RTP [5]. In fact, the RTP $q$-comb transport model [6], developed to describe the RTP electron transport barriers near rational $q$ surfaces, also models well the TEXTOR discharges with central ECRH. Heating just outside $q=1$ is shown to stabilize sawteeth. ECRH has also been applied during the initial current ramp-up in an attempt to better exploit the electron transport barriers. Pre-heating by counter-NBI is applied to try to establish a negative central shear (NCS) profile. Although central reversed shear does not appear to be achieved, ECRH in this current ramp phase with early heating from NBI reveals up to two internal electron transport barriers. Finally, the results of ECRH in RI mode are presented. A central transport barrier is found to prevent the usual degradation of confinement with additional central ECRH. As the waves are injected in a well focused beam, efficient ECRH is obtained right up to the cut-off density for the second harmonic $\mathrm{X}$-mode. The final section 4 provides a summary of the results and gives an outlook at the increased possibilities as soon as the new $140 \mathrm{GHz}$ system will become available.

\section{The 110 and $140 \mathrm{GHz}$ ECW systems}

In 1999 , the $500 \mathrm{~kW}, 200 \mathrm{~ms}, 110 \mathrm{GHz}$ gyrotron that had been used on the RTP tokamak $[7,8]$, was installed on TEXTOR. It has been operated for ECRH and CTS experiments during all of 2000 and the first few months of 2001. During the current shutdown for installation of the DED [1], the ECW system is extended with a new gyrotron, which should become operational at the restart of TEXTOR. The gyrotron was specified to generate $>800 \mathrm{~kW}$ in a Gaussian beam at pulse lengths $>3 \mathrm{~s}$, sufficient to reach steady state with current drive experiments. In recent tests a pulse length of $10 \mathrm{~s}$ has been achieved. The ECW systems are also described in $[9,10]$.

\subsection{Gyrotron and auxiliary supplies}

Both gyrotrons have been manufactured by Gycom, Russia. The $110 \mathrm{GHz}$ gyrotron, is a diode type gyrotron with nominal specifications of $500 \mathrm{~kW}$ power and $200 \mathrm{~ms}$ pulse length. It has achieved an electronic efficiency of $35 \%$. The cavity mode is converted to a Gaussian beam with better than $95 \%$ efficiency with the non-Gaussian content being absorbed near the gyrotron. For the experiments reported in this paper the power injected into the plasma is estimated at $270 \pm 30 \mathrm{~kW}$.

The new $140 \mathrm{GHz}$ gyrotron is also a diode type gyrotron without a depressed collector. At the factory test in April 2002, an output level of $0.85 \mathrm{MW}$ in the Gaussian beam has been demonstrated at $3.5 \mathrm{~s}$ pulse length and $1 \%$ duty cycle. The Gaussian beam is $97 \%$ pure ( $>99 \%$ after special matching optics). An electronic efficiency of $38 \%$ has been determined for these parameters. The frequency is stable to within $\approx 30 \mathrm{MHz}$. The gyrotron is a full $\mathrm{CW}$ design and during the factory test the pulse length has been limited by the load rather than the gyrotron. The dummy load at the TEXTOR site has been found to be capable of handling full $10 \mathrm{~s}$ pulses, which has allowed gyrotron tests up to the maximum pulse length set by the power supply. Since for some applications fast modulated operation of the power is required, the output power has been determined as a function of beam voltage. Continuous variation of the output power from $0.85 \mathrm{MW}$ at $71 \mathrm{kV}$ to $0.15 \mathrm{MW}$ at $57 \mathrm{kV}$ has been demonstrated with a frequency difference of only $70 \mathrm{MHz}$. The collector has been able to withstand the full pulse length, even at the reduced efficiency. Nevertheless, the heat load on the collector is quite 
high and is spread by sweeping of the beam using a collector coil with triangular, $10 \mathrm{~Hz}$, waveform. A four quadrant power supply with protection against faults was developed for this purpose. As the collector would also be at risk if the RF output becomes low at high beam voltage and current ('idling', e.g. a wrong cavity mode), a protection has been included to switch off if this condition occurs for longer than $50 \mathrm{~ms}$ during a (continuous or modulated) pulse.

The old high voltage supply, consisting of a transformer/ rectifier unit fed from the $10 \mathrm{kV}$ grid and a modulator/regulator unit, has been upgraded to $80 \mathrm{kV}, 50 \mathrm{~A}, 10 \mathrm{~s}$ supply. This required a major overhaul, replacement of critical components and installation of high pressure water cooling on the modulator tetrode. The power supply is equipped with many internal hard wired protections and since the protection levels are different for the two gyrotrons, a switch between 110 and $140 \mathrm{GHz}$ gyrotron operation now automatically switches the protection levels.

Of course, the cooling requirements for the $140 \mathrm{GHz}$ gyrotron are much more demanding than for the old gyrotron. Whereas the $110 \mathrm{GHz}$ gyrotron could be cooled using the spare capacity of the ICRH cooling system, two new cooling systems have been installed for the new gyrotron: one, a high pressure ( 8 bar), low flow (for cavity and tetrode) system and the other, a low pressure, high flow (1500 litre $\mathrm{min}^{-1}$ ) (for the rest) cooling system. Since there was little spare capacity on the primary cooling side available, primary cooling can automatically switch from the cooling tower to Rur (the local river) to drinking water, depending on the temperature. The gyrotron window (CVD diamond) has its own dedicated cooling circuit.

\subsection{Transmission line}

A quasi-optical line ( $\sim 25 \mathrm{~m}$ length) is used for transmission of the microwaves from gyrotrons to the tokamak. This line has already been used with the $110 \mathrm{GHz}$ gyrotron and has never given problems (albeit at the low power level of the old gyrotron); nor has it needed adjustment after the initial alignment. The gyrotrons each have their own beam forming (matching optics unit, MOU) and polarizer (MTP_using two rotatable grooved mirrors) units, but after those the same line is used for both gyrotrons. Since at the stage of the design of the transmission line, there was a possibility that it would in future be used with a source with variable frequency (FEM), the main part of the transmission line is confocal. It is therefore suitable for transmission of both frequencies. The aluminium mirrors are generally large enough to allow full pulse length transmission without the need for cooling. Some smaller mirrors, near the $140 \mathrm{GHz}$ gyrotron (some $\mathrm{Al}$ and some $\mathrm{Cu}$ ) are water cooled. The transmission line is designed to transmit two beams simultaneously: the beams would overlap at the torus window or could be separated at a waist position before the window if two launchers were available. Since the two gyrotrons cannot presently be operated simultaneously, this feature is not used. Instead, the beams are transmitted down the centre of the mirrors and a (interlocked) switchable mirror is used to transmit the microwaves from one or the other gyrotron. RF screening of the line is realized with an aluminium enclosure. Stray radiation is absorbed by water: at strategic positions inside the enclosure, Teflon hoses with low flow water cooling are applied. Before the installation of the $140 \mathrm{GHz}$ gyrotron, screening has only been applied outside the torus hall but with the increased transmitted energy the line will now be screened everywhere.

The transmission line enters the torus hall via a dog-leg or labyrinth erected on top of the torus hall entrance labyrinth. This labyrinth is lined with bricks and also serves as a long pulse (non-calorimetric) power dump. The exit of the dogleg to the torus hall is equipped with a shutter. When this shutter is closed and the power therefore dumped inside the dog-leg, operation of the gyrotron independent of the TEXTOR tokamak is possible (e.g. for tests, or commissioning). Calorimetric power measurements are performed using a small waterload that absorbs a small fraction $(\sim 1 \%)$ of the power, which is split off from the main RF beam using a grating on one of the mirrors in the MTP.

Presently, the transmission line includes a focusingdefocusing mirror set, mounted near the torus end of the transmission line, with variable distance between the mirrors. This makes it possible to vary the beam radius in the plasma centre by a factor of 3 , figure 2 . The beam waist position in the plasma can also be displaced using this (motorized) construction (e.g. to have a minimum size waist near the inner wall, where the DED perturbation is largest).

The torus window is presently a large diameter $(\varnothing=$ $180 \mathrm{~mm}$ clear view) water-free fused silica window that is resonant at both 110 and $140 \mathrm{GHz}$. Face air cooling is used. This window limits the pulse length in case of the $140 \mathrm{GHz}$ gyrotron to $1-2 \mathrm{~s}$ (to be determined), and the duty cycle to less than $0.1 \%$. The window will be replaced by a CVD diamond window (resonant at $140 \mathrm{GHz}$ ) in 2003 when it becomes the limiting component for the pulse length (i.e. after replacement of the launcher, see below). The window temperature is monitored using an IR thermometer. The window is mounted slightly oblique to the beam to direct reflected power into a dump.

\subsection{Launcher}

The launcher used with the $110 \mathrm{GHz}$ gyrotron consists of a fixed focusing mirror (copper) and a steerable flat mirror

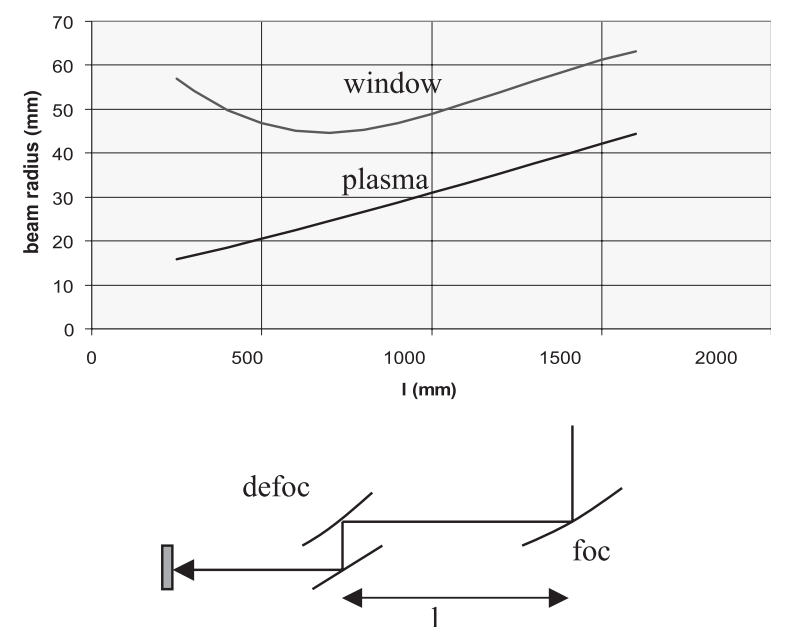

Figure 2. Beam radius (at $1 / e$ value of the $E$-field) at the window and in the plasma centre as a function of the distance between the focusing and defocusing mirrors. 


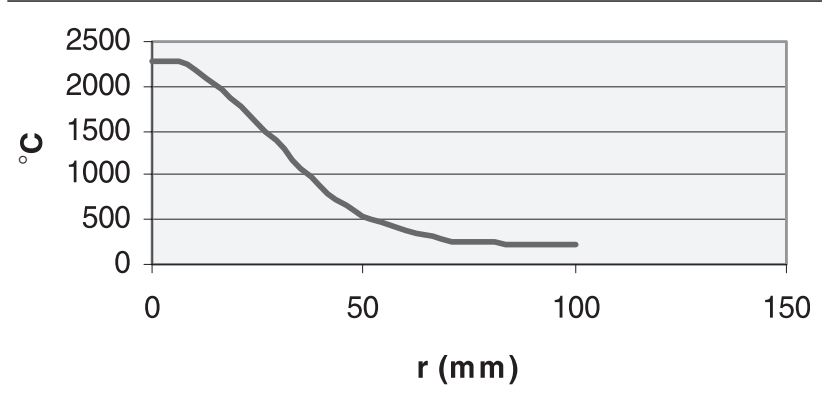

Figure 3. Surface temperature of a stainless steel mirror as a function of radius after a $3 \mathrm{~s}$ pulse. Increased surface resistivity (3\% loss), initial temperature $200^{\circ} \mathrm{C}$ and no change of parameters with temperature have been assumed for this illustration.

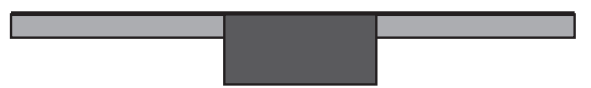

Figure 4. Preliminary steerable mirror with a central copper heat sink.

(stainless steel). The steerable mirror can be rotated around a vertical axis to choose the angle of injection in the toroidal direction between $-45^{\circ}$ and $+45^{\circ}$. It can also be rotated around a horizontal axis (between $-15^{\circ}$ and $+15^{\circ}$, which means that the beam injection angle varies between $-30^{\circ}$ and $+30^{\circ}$ ) to cover the poloidal cross section. The size of the mirrors is the maximum size that could be fitted inside the port ( $\sim 200 \mathrm{~mm}$ width), to obtain maximum beam focusing in the plasma. Stainless steel was used for the steerable mirror to limit forces due to eddy currents during a disruption. The present steering mechanism is not capable of dealing with these forces if a copper mirror were used. After the first experimental campaign it was noticed that the surface resistivity of the steerable mirror had further increased (to give about $3 \%$ loss) due to boronization. This type of mirror is unsuitable with the increased power level and pulse length of the new gyrotron. To illustrate this, the calculated surface temperature is given in figure 3 (assuming no change of parameters with temperature-in reality the situation would be worse).

Although a copper mirror would suffer the same increased surface resistivity, the surface temperature would remain limited (to $<600^{\circ} \mathrm{C}$ ) due to the high heat conductivity. However, to use the full pulse length capability, the mirror should either be cooled or protected from deposition during boronization and other deposition processes. As a preliminary measure the existing launcher will be used, however, with a copper coating and a central copper heat sink (only central to limit disruption forces), figure 4 . In the meantime a new launcher is being developed that will be capable of withstanding disruption forces using a full copper or molybdenum mirror and which can be retracted during boronization and other deposition processes, figure 5 .

When this launcher is installed, the torus window will also be replaced with a CVD diamond disc. Since the diameter of available discs is limited, the fixed mirror will be replaced by a defocusing mirror while the steerable mirror will become focusing to still obtain a minimum beam size in the centre of the plasma.

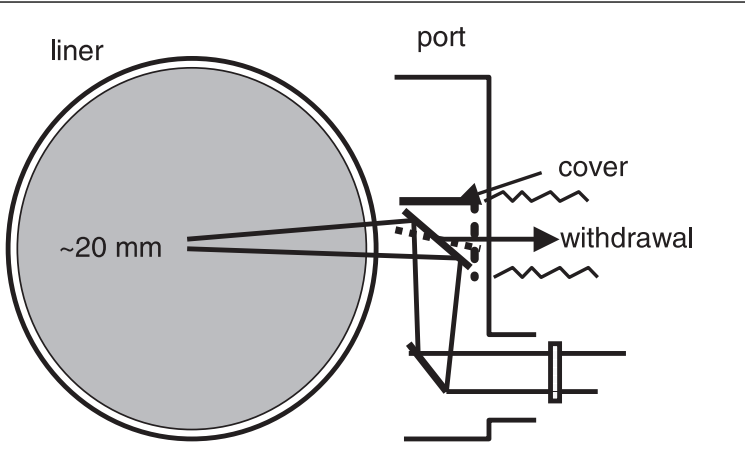

Figure 5. Sketch of the new launcher under development. It can be retracted and covered when not in use to limit surface deposition.

\subsection{Control}

With the introduction of an additional gyrotron, the interlock and protection systems have been extended significantly. Since operation can be switched from one gyrotron to the other, interlocks have been introduced to ensure that all relevant protection parameters are switched automatically. A schematic overview of the control system is provided in figure 6 .

Additional protection features were required. As an example, firing into an empty torus or a plasma with insufficient absorption is no longer permissible with the increased pulse length of the $140 \mathrm{GHz}$ gyrotron and a protection unit has developed to prevent this (with limited overriding possibilities for pre-ionization experiments). All important protection features are hardwired, but they are interfaced to a programmable logic controller (PLC). The PLC also provides the interface to the installation for remote control from a PC in the TEXTOR control room. Both manual (protected) operation and full remote operation are possible.

The data acquisition system is VME based and records some diagnostic signals as a function of time (e.g. power, frequency, etc) as well as the settings of the installation (e.g. launcher angle, polarization etc). The data are transferred to a central storage facility and can be assessed using a newly developed protocol, which can be used to access all TEXTOR data [11]. All control software was re-written. Although the PLC allows convenient control of the installation, it is slow. For fast feedback purposes on the basis of diagnostic signals it is therefore necessary to have direct access to timing of the gyrotron pulses (e.g. for mode stabilization or DED diagnostic purposes) and launcher angle (e.g. to track the mode position as it changes with time). The possibilities for this direct control are already provided in the present control system. They will be the subject of further developments.

\section{Experimental results with the preliminary $110 \mathrm{GHz}$ ECW system}

Although the power and pulse length of the preliminary $110 \mathrm{GHz}$ system are limited, the good localization of the ECRH has nevertheless given rise to quite a number of very interesting results. In particular, results are reported below on central ECRH during the current flat top of Ohmic discharges, which confirm the existence of a central electron transport barrier. This barrier is located near the $q=1$ surface. With ECRH, during the current ramp phase, up to two simultaneous electron 


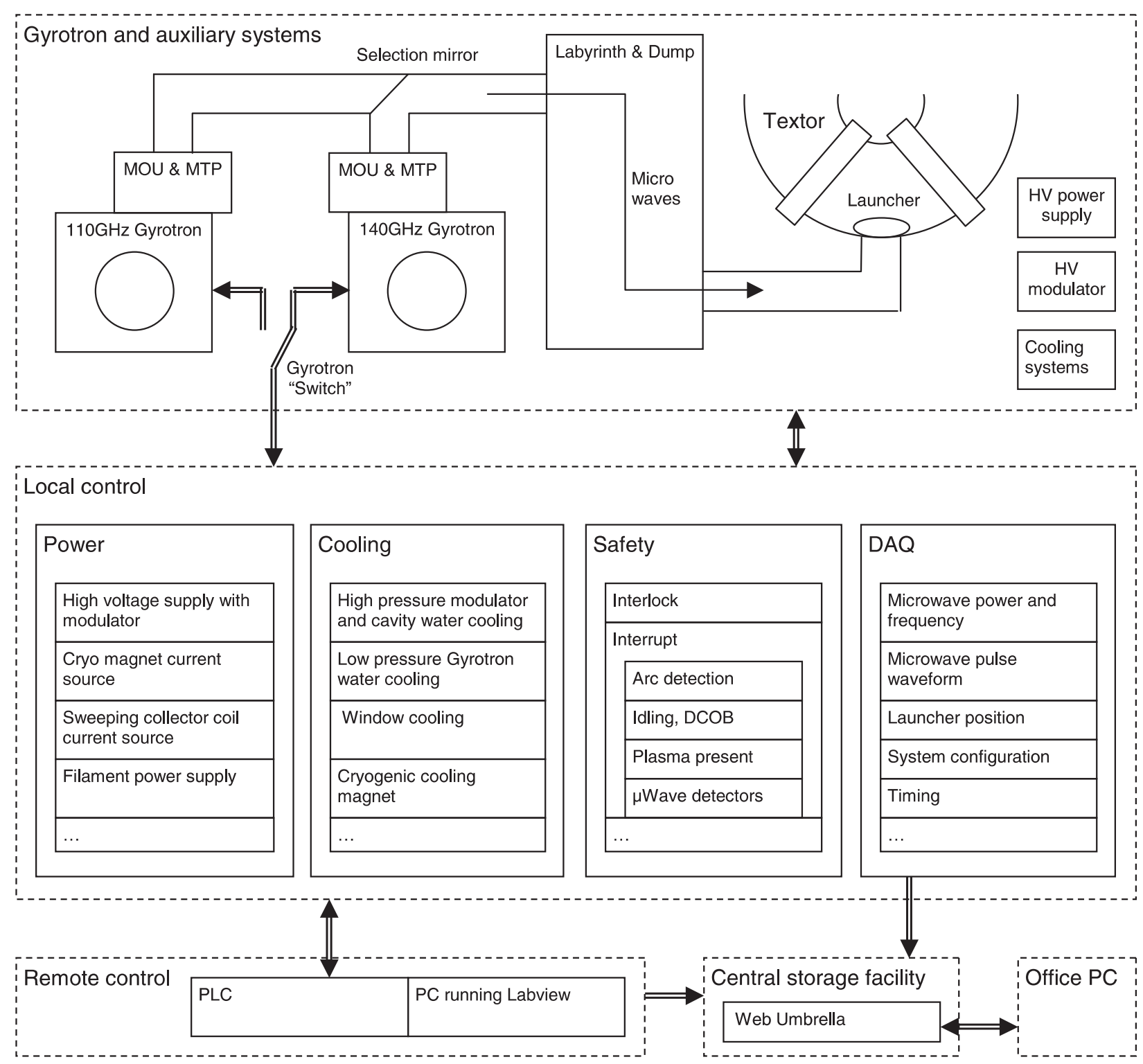

Figure 6. Schematic overview of the control system. The top section represents the equipment, the central section shows the associated control circuits together with the master unit, and the bottom section shows the computer systems for remote control and data acquisition.

transport barriers are observed. Finally, ECRH is proven to be especially effective during the RI mode, indicating the presence of a central electron transport barrier during the RI mode as well.

\subsection{Central ECRH: the $q=1$ transport barrier and sawtooth control}

The dominance of ECRH power in RTP led to a highly nonlinear response of the plasma profiles to changes in ECRH localization. This suggested the presence of multiple electron transport barriers located near the low rational values of the safety factor $q$ [5]. To explain these results, the so-called $q$-comb model for electron heat conductivity, $\chi_{\mathrm{e}}$, has been developed in which $\chi_{\mathrm{e}}$ is supposed to be a function of $q$ only with a constant high value interspersed with narrow regions of low conductivity located near the low rational values of $q$ : $1, \frac{4}{3}, \frac{3}{2}, 2, \frac{5}{2}, 3, \frac{7}{2}, \ldots[6]$. When the absolute values of the heat conductivity are scaled by a factor of 0.83 consistent with L-mode scaling ([12] equation (6)) between RTP and TEXTOR, the same model also describes well the TEXTOR discharges with central ECRH [13,14], as shown in figure 7. However, large uncertainties in the temperature measurements from the high resolution TVTS system [2], when combined with the relatively moderate heat flux across $q=1$ with central ECRH in the present TEXTOR case, do not allow a direct confirmation of the central, $q=1$ transport barrier. Nevertheless, the collective action of the barriers at low rational $q$-surfaces describes the global profile shape and peakedness very well.

To better corroborate the RTP results on transport barriers, the ECRH deposition was scanned through the TEXTOR plasma by changing the toroidal magnetic field. This was done in Ohmic target plasmas at two different values of the plasma current: $I_{\mathrm{p}}=255 \mathrm{kA}$ (with $\bar{n}_{\mathrm{e}}=2.5 \times 10^{19} \mathrm{~m}^{-3}$ ) and $I_{\mathrm{p}}=357 \mathrm{kA}\left(\right.$ with $\left.\bar{n}_{\mathrm{e}}=1.9 \times 10^{19} \mathrm{~m}^{-3}\right)$. The $q=1$ barrier 


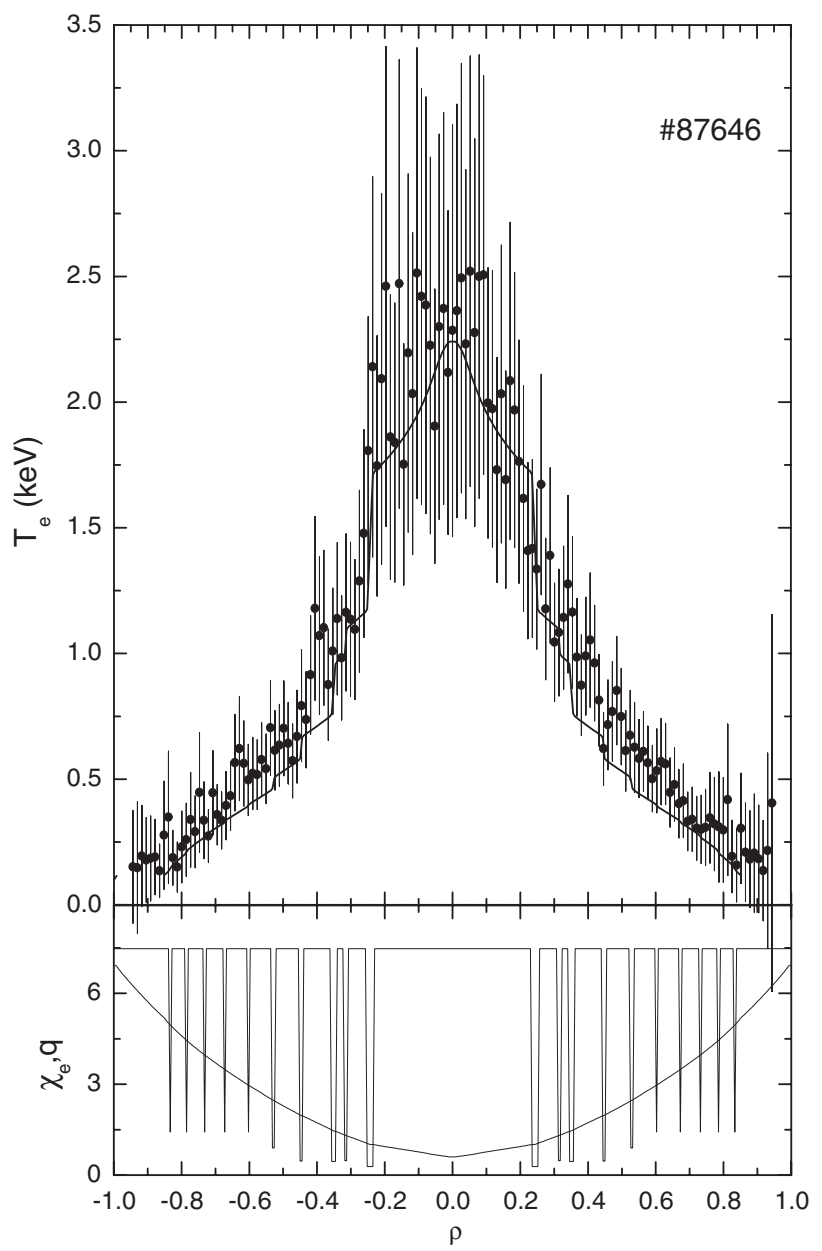

Figure 7. The $T_{\mathrm{e}}$ profile in a typical TEXTOR discharge with central $250 \mathrm{~kW}$ ECRH simulated with the RTP $q$-comb model for the electron heat conductivity. Dots represent measured $T_{\mathrm{e}}$. The curve gives the result of the simulation. The bottom panel shows the $q$ profile and the corresponding profile of the electron heat conductivity $\chi_{\mathrm{e}}$.

is seen in the data of either the central temperature (figure 8) or the total plasma energy. For higher current, the position of the barrier is at larger radius as expected from the larger $q=1$ radius. The relation between the barrier and $q=1$ is further confirmed by the sawtooth inversion radius, which is close to the barrier position.

It is worthwhile to have a close look at the sawtooth behaviour in these experiments. Most tokamaks equipped with ECRH report a lengthening of the sawtooth period or complete sawtooth stabilization by ECRH near the sawtooth inversion radius (see, e.g. the early experiments on T-10 [15] or the more recent and more detailed results from TCV [16]). These are corroborated by the present results: the sawtooth period has a marked maximum when ECRH is deposited close to the barrier and sawtooth inversion radius (see figure 9). For the lower current case, sawteeth are stabilized during the $200 \mathrm{~ms}$ ECRH pulse when the power is deposited in a relatively broad region outside the inversion radius. There is no observation of a density pump out upon application of ECRH when the sawteeth are stabilized. Once sawteeth are stabilized by offaxis ECRH, the discharge remains in a non-sawtoothing regime

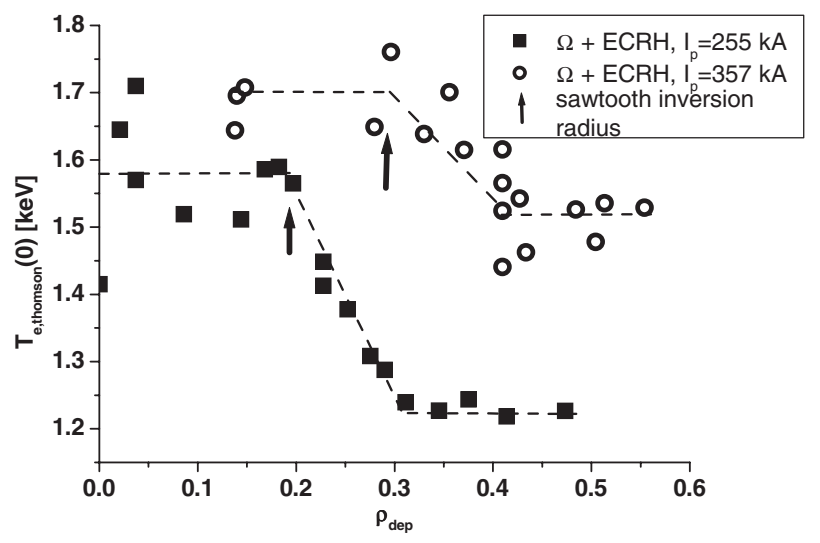

Figure 8. The central $T_{\mathrm{e}}$ achieved in Ohmic discharges with ECRH as a function of the ECRH deposition radius $\rho_{\text {dep }}$. The ECRH deposition radius is varied by changing the toroidal field. Two datasets are shown: squares for $I_{\mathrm{p}}=255 \mathrm{kA}$ and circles for $357 \mathrm{kA}$. A distinct drop in central temperature is observed, when ECRH is deposited outside the sawtooth inversion radius (indicated by arrows).

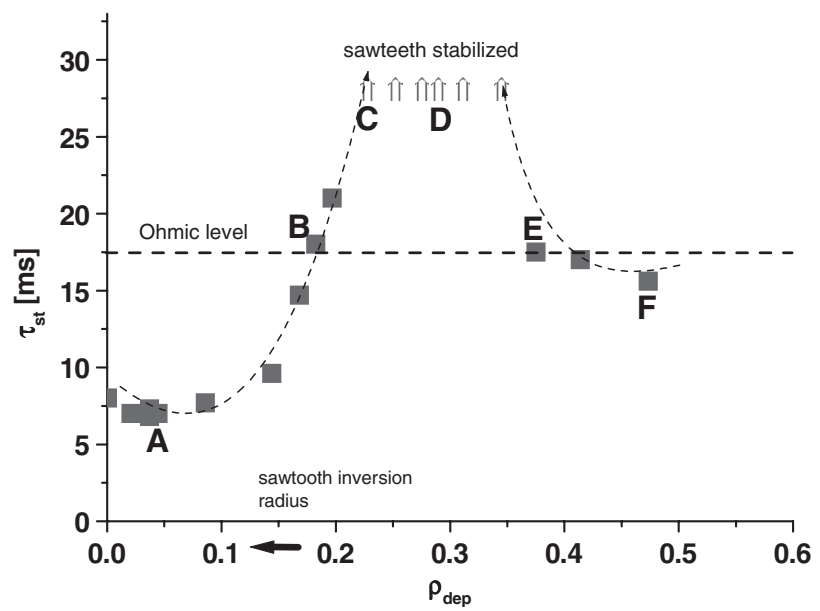

Figure 9. The corresponding sawtooth for the Ohmic discharges in figure 8 as a function of the ECRH deposition radius for

$I_{\mathrm{p}}=255 \mathrm{kA}$. The sawteeth are stabilized, when ECRH is deposited within a region just outside the sawtooth inversion radius. The characters A-F refer to the discharges depicted in figure 10.

after switch-off of the ECRH. This behaviour is depicted in figure 10 representing the central soft x-ray (SXR) emission for different EC deposition radii. Although the density and temperature are seen to return to their pre-ECRH values, the SXR intensity remains high and may increase even further after ECRH. This points to impurity accumulation and an increased $Z_{\text {eff }}$ preventing the central current to peak again after ECRH.

For heating at or just outside the inversion radius, sawteeth are marginally stabilized. An initial increase in SXR emission indicates a rise in central impurity content and a delay in the sawtooth crash. After the first crash, however, the impurities are ejected after which the discharge returns to the normal sawtoothing regime.

3.1.1. Modulated electron cyclotron heating (MECH). Electron heat transport was further scrutinized with modulated ECRH (MECH) experiments. We report here on the analysis 


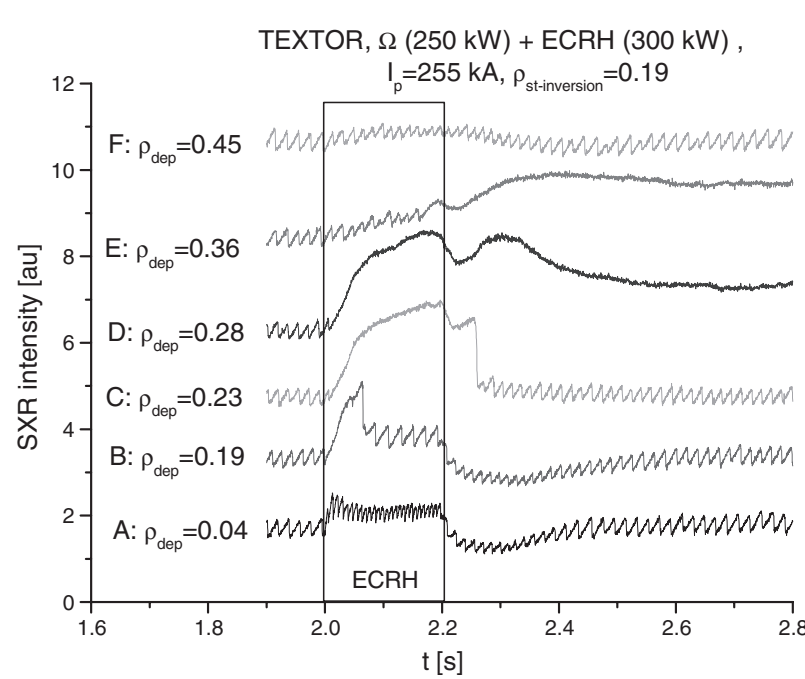

Figure 10. Time traces of the SXR intensity for discharges differing in EC deposition radius. For central deposition (A, see figure 9) the sawtooth period is shortened. For heating outside the inversion radius $(\mathrm{C}-\mathrm{E})$ sawteeth are stabilized. The discharges remain in the non-sawtoothing regime even after switch-off of the ECRH. For D and E the SXR intensity is about a factor of 2 higher than in the Ohmic case before the ECRH. Note the crashes in B and C after which the discharges return to the normal sawtoothing regime.

of a series of similar discharges with $\mathrm{MECH}$ in which the ECH power deposition radius $\left(\rho_{\text {dep }}\right)$ was scanned by small changes of the toroidal magnetic field $\left(B_{\mathrm{t}}\right)$. In these discharges $\left(I_{\mathrm{p}}=350 \mathrm{kA}, q_{\mathrm{a}} \simeq 4, \bar{n}_{\mathrm{e}} \approx 2 \times 10^{19} \mathrm{~m}^{-3}\right)$ eight cycles of MECH were applied, at frequency $f=33.3 \mathrm{~Hz}$ and duty cycle $d_{\mathrm{c}}=0.8$.

The second harmonic ECE $T_{\mathrm{e}}$ signals are Fourier analysed to calculate phases $(\phi)$ and amplitudes $(A)$ relative to the MECH pulse. Figure 11 shows the first harmonic phase and amplitude profiles of five TEXTOR discharges of the scan described above (upper and lower panels, respectively); the discharges are indicated by different plot symbols; $\rho_{\text {dep }}$ for each discharge is indicated by a vertical arrow in the upper plot. The rise of amplitudes outside $\rho \simeq 0.6$ on the HFS is most likely due to overlap with the third harmonic ECE-emission, and is therefore neglected in the analysis. The following observations can be made from figure 11:

(i) A change of $\rho_{\text {dep }}$ from nearly central (circles) to $\simeq 0.24$ (diamonds) is seen to cause only modest changes in both $\phi$ and $A$ profiles.

(ii) A modest change of $\rho_{\text {dep }}$ from $\simeq 0.24$ to $\simeq 0.36$ is seen to cause a sharp transition from a $\phi$ profile with a low central value to a $\phi$ profile with a high central value with clear off-axis minimum (squares and plus signs).

(iii) During this transition, the $A$ profile remains peaked at first (squares), with only a gradual decrease of the central value; only for larger $\rho_{\text {dep }}$ the $A$ profile develops an offaxis maximum.

If one translates these observations into a characterization of the electron heat diffusion coefficient $\left(\chi_{\mathrm{e}}\right)$, the following picture arises: (i) $\chi_{\mathrm{e}}$ is high inside $\rho \simeq 0.24$; (ii) there is a low heat transport or even a transport barrier in the region between $\rho \simeq 0.24$ and 0.36 ; (iii) the fact that the $A$ profile does not change in parallel with the strong change of the $\phi$ profile

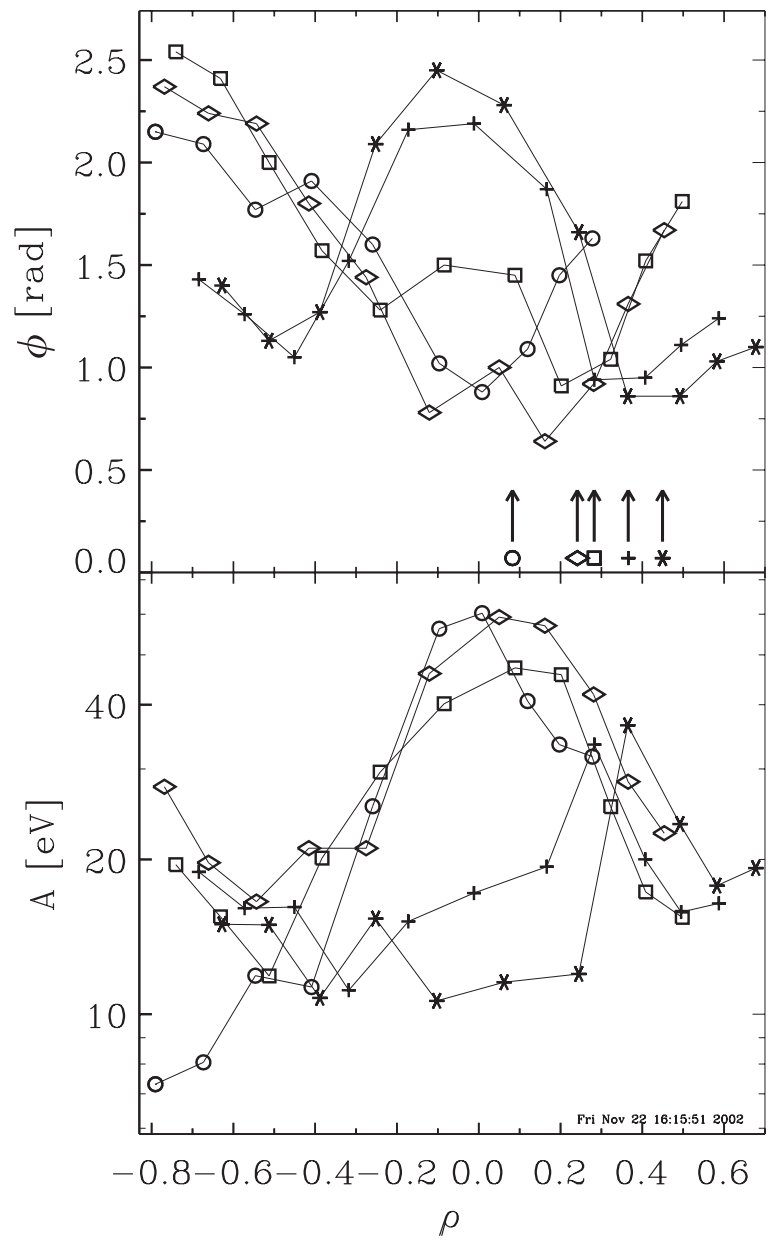

Figure 11. First harmonic phase (upper) and amplitude (lower panel) profiles of five similar TEXTOR discharges in which $\rho_{\text {dep }}$ (indicated by vertical arrows in the upper plot) was scanned. Plasma parameters: $I_{\mathrm{p}}=350 \mathrm{kA}, q_{\mathrm{a}} \simeq 4, \bar{n}_{\mathrm{e}} \approx 2 \times 10^{19} \mathrm{~m}^{-3}$.

when $\rho_{\text {dep }}$ is moved over this narrow region, indicates that this apparent barrier, in part, might be caused by local inward heat convection instead of a low $\chi_{\mathrm{e}}$, as was seen before on RTP [17]. The value for the central $\chi_{\mathrm{e}}$ might be related to the presence of sawteeth, which for these discharges have a frequency of $\sim 100 \mathrm{~Hz}$, i.e. higher than the modulation frequency.

These qualitative statements are further analysed in figure 12, where the first harmonic central phases and amplitudes of the full dataset at $350 \mathrm{kA}$ are plotted, together with model calculations. The $\chi_{\mathrm{e}}$ profiles used in the simulations are given in figure 13. First, a simple diffusive model is tried with a parabolic shape of $\chi_{\mathrm{e}}$ (dotted lines, assuming $\chi_{\mathrm{e}}(0)=1.5$ and $\left.\chi_{\mathrm{e}}(a)=6 \mathrm{~m}^{2} \mathrm{~s}^{-1}\right)$; this clearly does not represent the experimental findings. As a first improvement, $\chi_{\mathrm{e}}$ is supposed to be high $\left(20 \mathrm{~m}^{2} \mathrm{~s}^{-1}\right.$; dashed lines) inside the region $\rho<0.24$; this represents the data with small $\rho_{\text {dep }}$ much better, however, it does not reflect the decrease of slope of $\phi$ outside $\rho \simeq 0.4$. Therefore, as a further improvement, a local barrier in the region $0.24<\rho<0.36$ with $\chi_{\mathrm{e}}=0.2 \mathrm{~m}^{2} \mathrm{~s}^{-1}$ is added (dashed-dotted lines); this yields a better simulation of the central phases; however, it overestimates the decrease of the central amplitudes outside $\rho \simeq 0.24$. Hence, as the final step, a localized region with 


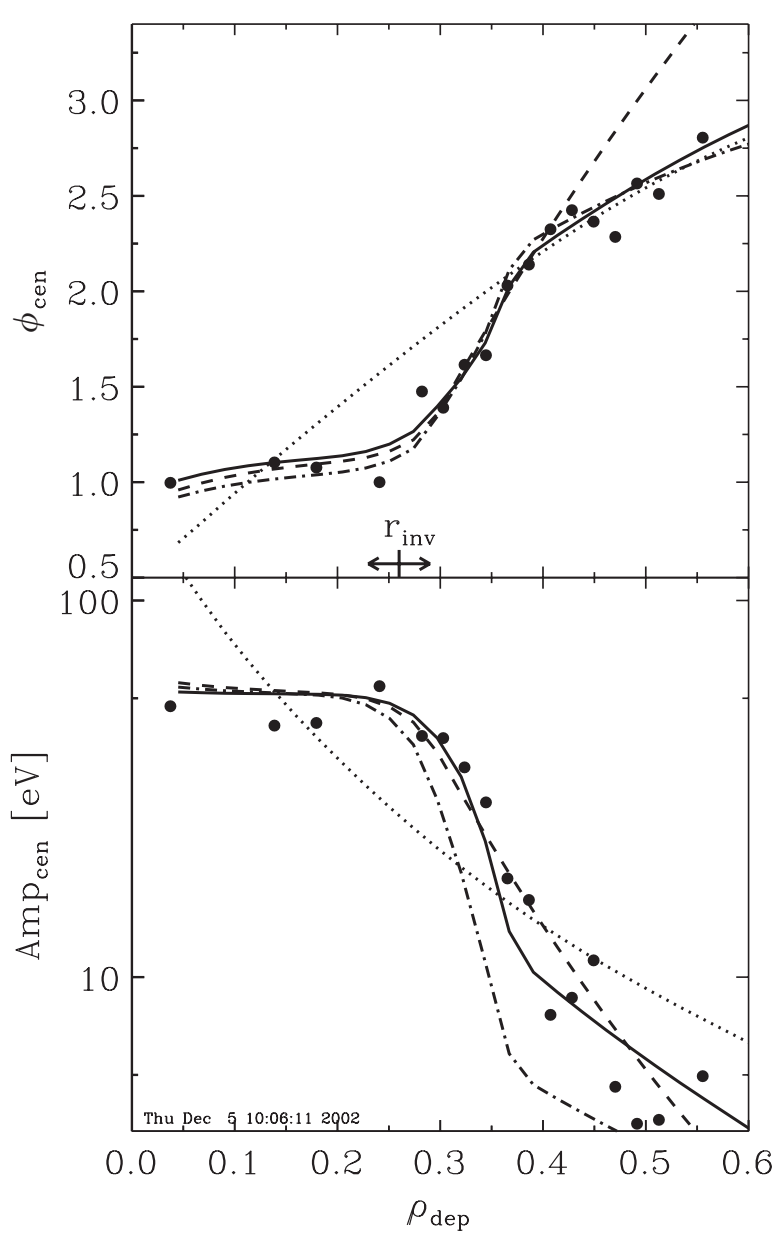

Figure 12. Central phases (upper) and amplitudes (lower panel) of the full dataset at $350 \mathrm{kA}$, together with simulations of central phases and amplitudes with four different models (see text). The sawtooth inversion radius $\left(r_{\text {inv }}\right)$, including its uncertainty, is indicated as well.

inward heat convection around $\rho=0.3$ was also added (full lines); this further improves the simulations.

The sawtooth inversion radius $\left(r_{\text {inv }}\right)$ is indicated in figure 12 as well. The barrier is seen to lie just outside $r_{\text {inv }}$, and thus confirms the steady state data (figure 8). As indicated before, the barrier might partially be due to inward heat convection instead of just low thermal diffusion; however, the quality of the available data does not allow a firm conclusion in that direction.

The role of low order rational $q$-values in the formation of internal barriers has been observed on many other tokamaks (for example, JT-60U [18], JET [19] and T-10 [20]). These experiments all required either low (optimized) magnetic shear or reversed magnetic shear (see also the review paper [21]) and in most of the cases the rational $q$-value played a decisive role only in triggering the barrier. The $q=1$ barrier presented here, however, occurs for the natural positive magnetic shear in a plasma with only a modest velocity shear. Neither a power threshold for the formation (although the minimum applied ECRH power was still $200 \mathrm{~kW}$ ) nor any triggering event could be identified. The clear correlation of the barrier with the sawtooth inversion radius suggests that for these discharges the value of safety factor $q$, rather than its shear, is the determining factor for the presence of the barrier.

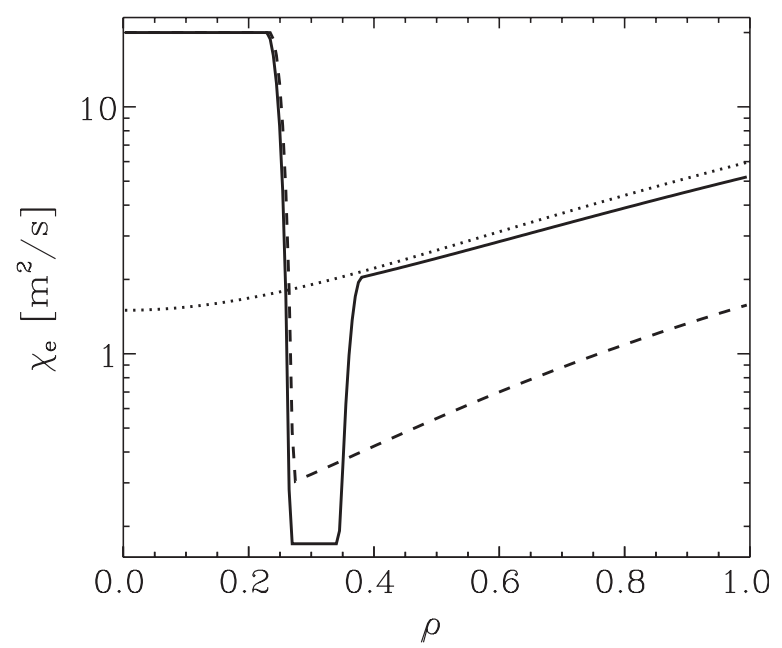

Figure 13. The electron heat conductivities as used in the simulations of the heat pulse propagation. The line types correspond to those showing the simulation results in figure 12 . Note that the dashed-dotted and full lines overlap, as they differ only in the inward convection velocity, which for the full line is $v_{\text {conv }}=17 \exp \left(-(\rho-0.3)^{2} / 0.08^{2}\right) \mathrm{m} \mathrm{s}^{-1}$.

\subsection{ECRH during the current ramp phase}

Given that the electron heat conductivity displays barriers near the rational values of $q$, it will be attractive to control the current density profile in such a way as to make optimal use of these barriers. One way to optimize the $q$ profile is by creating NCS, i.e. a hollow current density profile, or at least very low central shear. Both in ASDEX-Upgrade as well as in TCV a strong electron transport barrier and high $T_{\mathrm{e}}(0)$ have been obtained in discharges with central counterECCD [22,23]. In these experiments, the counter-ECCD is instrumental both for sustaining or achieving NCS as well as for supplying the central electron heating. The available $270 \mathrm{~kW}$ of the preliminary $110 \mathrm{GHz}$ ECRH system, was insufficient to achieve a central shear reversal in TEXTOR and, consequently, another technique had to be used to create NCS.

A generally used technique to create a hollow current density profile is by means of early heating during the current rise phase of the discharge: an increased temperature slows down current penetration, resulting in a hollow current profile $[21,24]$. This method has been employed in a series of TEXTOR discharges with the aim of exploring a possible NCS regime [25]. In the experiments, a fast initial ramp (100 ms) up to $I_{\mathrm{p}}=200 \mathrm{kA}$ is followed by a slower ramp up to $350 \mathrm{kA}$ at $t=600 \mathrm{~ms}$. Early heating is provided by $1 \mathrm{MW}$ of counterNBI from $t=200 \mathrm{~ms}$. In addition to providing the required plasma heating, a central counter driven current was expected to assist in the attainment of NCS. Somewhat later, ECRH $(270 \mathrm{~kW}, t=250-450 \mathrm{~ms})$ is applied for additional electron heating to highlight the presence of electron transport barriers. Midway during the ECRH pulse, at $t=350 \mathrm{~ms}$, the electron temperature profile is measured by Thomson scattering (TS).

The evidence from polarimetry just before and after the ECRH, as well as from current diffusion calculations including the beam driven currents indicates that the central shear in this phase is at best marginally reversed or flat. The central and/or minimum $q$ value just before ECRH is in 
the range of 1.5-2.5. Unfortunately, no polarimetry data are available during ECRH as a consequence of perturbations to the measurements from the microwaves. In particular, during central ECRH, experimental results indicate a fast evolution of the central current profile with $q$ dropping below 1 near the end of the ECRH pulse as evidenced by the start of sawtoothing. In normal discharges without early heating, sawteeth generally appear only much later. In spite of the higher temperature, the strong central peaking of the $T_{\mathrm{e}}$ profile leads to a faster central current penetration, because of the reduced gradient length. This is confirmed by the current diffusion calculations using the ASTRA code.

In conclusion, a NCS regime has not been established, but discharges with a broad low shear region in the centre have been obtained. Nevertheless, the results on ECRH in this current ramp phase of the discharge has produced a number of striking results. The $T_{\mathrm{e}}$ profiles as obtained for different positions of ECRH deposition are shown in figure 14. Two electron transport barriers are identified in these profiles at normalized minor radii of $\rho=0.13$ and 0.35 . The region inside the inner barrier is much larger than the width of the ECRH deposition profile (typically $\Delta \rho=0.05$ ). The lack of polarimetry data during ECRH makes it impossible to link these barriers to specific values of $q$.

Apart from providing the necessary pre-heating, counterNBI also proved essential for stability during central ECRH. Without NBI, ECRH in the current ramp phase is accompanied by one or more strong core collapses. Core collapses are also observed with off-axis ECRH as seen in the central $T_{\mathrm{e}}$ evolution (figure 15). A particularly strong core collapse is observed in the case where EC deposition coincides with the inner barrier (discharge no 89940).

Replacing part or all of counter-NBI by co-NBI leads to increased MHD activity during ECRH as shown in figure 16. Even when only part of the counter-NBI is replaced by co-NBI, the discharge evolution is strongly affected: increased MHD activity appears to prevent central current penetration, such that central $q=1$ and sawteeth are never attained. Instead, the discharge typically ends in a disruption some time after the ECRH. Only the combination of early heating with counterNBI plus a subsequent pulse of central ECRH is seen to result in an evolution towards a stable sawtoothing discharge. Current diffusion calculations confirm that only with the combination of counter-NBI and central ECRH the central $q$ value drops below 1 near the end of the ECRH pulse. With either balanced or co-NBI the current density profile remains significantly broader. This might explain the susceptibility of these discharges to, for example, the $m=2, n=1$ tearing mode, which in the subsequent evolution plays a major role finally ending in a disruption. The differences in rotation between these discharges (figure 17) might also play a role in their stability. It is noted here that a later start of co-NBI, after or at the start of the current flat top phase, is an often used method at TEXTOR to prevent or suppress MHD $m=2$, $n=1$ modes [26].

\subsection{ECRH in the RI mode}

On TEXTOR, a regime with improved confinement, RI mode, has been established by injection of radiating species in the
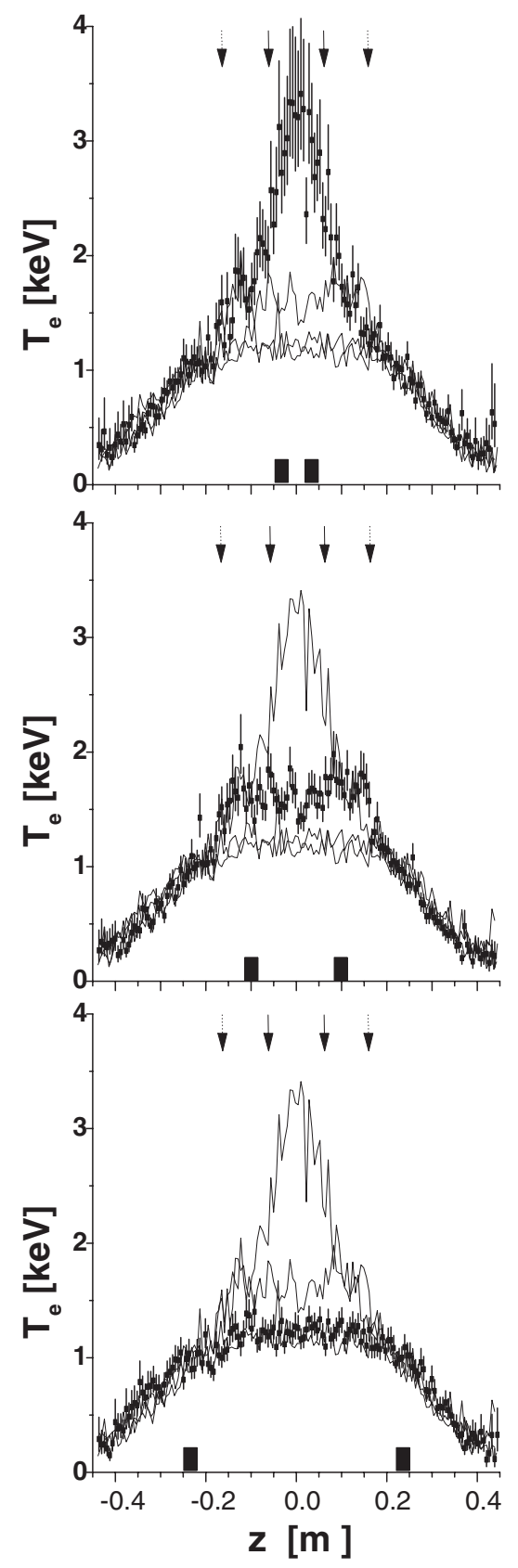

Figure 14. $T_{\mathrm{e}}$ profiles from TS at $t=350 \mathrm{~ms}$ for different positions of ECRH deposition. Each plot shows the measurements and their error bars for a particular discharge: 89932 (top)

$\rho_{\text {dep }}=r_{\text {dep }} / a=0.06 ; 89935$ (middle) $\rho_{\text {dep }}=0.23 ; 89939$ (bottom) $\rho_{\text {dep }}=0.50$. The other discharges (including 89933 with NBI-only) are represented in each plot by a thin line through the data points.

The arrows indicate the positions of the barriers and the horizontal bars indicate the ECRH deposition region.

plasma edge (typically $\mathrm{Ne}$ or $\mathrm{Ar}$ ) [3]. Apart from a radiating mantle, this regime is characterized by establishment of a peaked density profile with a possible suppression of ion temperature gradient (ITG) turbulence as a consequence [27]. Impurity seeding is also seen to lead to improved performance in DIII-D and JET [28]. The energy confinement in RI mode scales with line averaged density $\bar{n}_{\mathrm{e}}$ as in the linear Ohmic confinement regime, but the general degradation of 


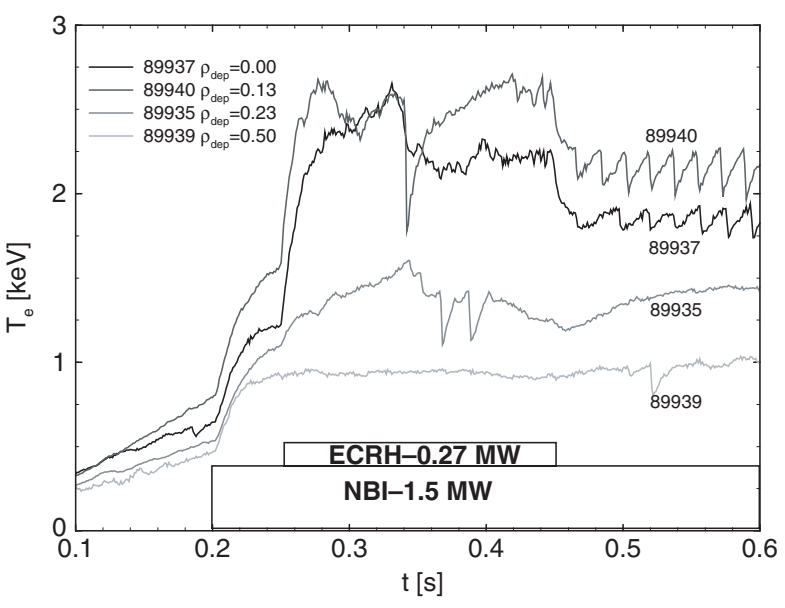

Figure 15. The evolution of $T_{\mathrm{e}}(0)$ from ECE for different positions of the ECRH deposition: $89937 \rho_{\text {dep }}=0.00 ; 89940 \rho_{\text {dep }}=0.13$; $89935 \rho_{\text {dep }}=0.23 ; 89939 \rho_{\text {dep }}=0.50$.

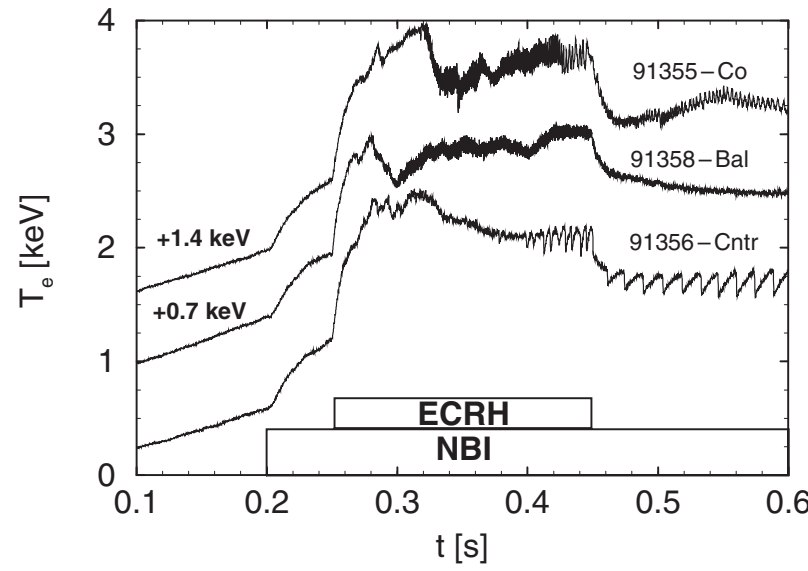

Figure 16. The evolution of $T_{\mathrm{e}}(0)$ from ECE for different ratios of counter- and co-NBI: 91356 counter-NBI; 91358 balanced NBI; 91355 co-NBI.

confinement with total heating power remains [3]:

$$
\tau_{\mathrm{RI}} \propto \bar{n}_{\mathrm{e}} P_{\mathrm{tot}}^{-2 / 3} .
$$

The improvement of confinement is attributed to the suppression of the ITG turbulence, which is a major cause of anomalous ion heat conductivity. The electron heat conductivity and its improvement in RI mode are less wellknown. It is thus of particular interest to study pure electron heating as provided by ECRH. However, the $200 \mathrm{~ms}$ pulse length and $270 \mathrm{~kW}$ injected power of the preliminary $110 \mathrm{GHz}$ system are limited when compared to confinement times of about $50 \mathrm{~ms}$ and the total heating power of about $2.7 \mathrm{MW}$ of typical RI mode discharges. Yet, interesting results have been obtained on ECRH in the RI mode [29].

The effect of central ECRH in a typical RI mode discharge is shown in figure 18, which gives the time evolution of the total heating power and the diamagnetic energy $\left(W_{\text {dia }}\right)$. Also shown is the energy content $W_{\mathrm{dia}, \mathrm{RI}}$ expected on the basis of RI mode confinement scaling. During ECRH the density continues to rise: no density pump-out is observed during ECRH in the RI mode. Given that $P_{\mathrm{ECRH}} / P_{\mathrm{tot}} \approx 0.27 / 2.7[\mathrm{MW}] \approx 10 \%$,

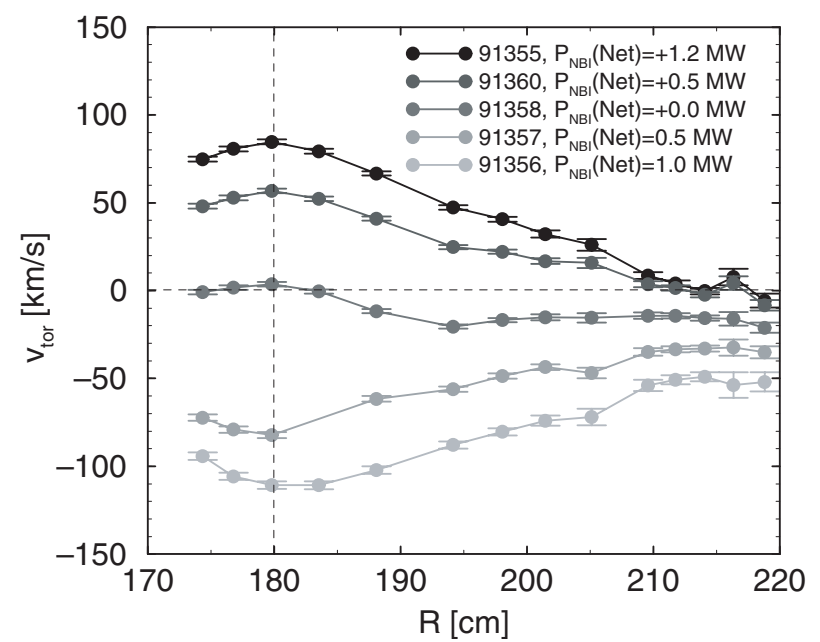

Figure 17. Profiles of rotation velocity (at $t=350-400 \mathrm{~ms}$ ) from CXRS for discharges with different ratios of co- and counter-NBI. For each discharge, the net current driving power $P_{\text {co }}-P_{\text {counter }}$ is quoted. The total NBI power in all cases is $1.2 \mathrm{MW}$

an increase in stored energy during ECRH of typically $3 \%$ is expected on the basis of the RI mode scaling. However, in many cases $\delta W_{\text {dia }} / W_{\text {dia }} \approx 9 \%$ is observed, i.e. the confinement degradation with total power does not appear to apply to ECRH. Figure 19 shows $\delta W_{\mathrm{dia}} / W_{\mathrm{dia}}$ normalized to $\delta P / P_{\text {tot }}$ for a number of discharges as a function of the line average density $\bar{n}_{\mathrm{e}}$. In comparable L-mode discharges (not shown in figure 19) the normalized efficacy $\left(\delta W_{\mathrm{dia}} / W_{\mathrm{dia}}\right) /\left(\delta P / P_{\mathrm{tot}}\right)$ is close to the expected value of 0.3. The effectiveness of ECRH is seen to hold up to $\bar{n}_{\mathrm{e}} \approx 5.5 \times 10^{19} \mathrm{~m}^{-3}$. Abel inversion of the interferometry data shows that this corresponds to $n_{\mathrm{e}}(0) \approx$ $7.5 \times 10^{19} \mathrm{~m}^{-3}$, coinciding within measurement accuracy with the $110 \mathrm{GHz} \mathrm{X}$-mode cut-off density. The central ECE channels start decreasing when $\bar{n}_{\mathrm{e}} \geqslant 4.3 \times 10^{19} \mathrm{~m}^{-3}$. That the effectiveness of ECRH is much less affected by the approach of the density cut-off than ECE can be understood by the differences in antenna patterns of the ECE and ECRH systems: whereas the ECRH wave beam is well focused inside the plasma, the ECE antenna pattern has a divergence of $12.5^{\circ}$ (1/e half width of the intensity). Ray tracing calculations with TORAY and NOTEC confirm that the ECRH power deposition profile is practically constant virtually up to the cut-off density, while the radiation temperature in the 109 and $112 \mathrm{GHz}$ central ECE channels starts decreasing already at $n_{\mathrm{e}}(0) \geqslant 6.5 \times 10^{19} \mathrm{~m}^{-3}$. Above the X-mode cut-off the efficiency of ECRH decreases below the $2 \%$ level. At these high densities, injecting O-mode yields a normalized efficacy of $\left(\delta W_{\mathrm{dia}} / W_{\mathrm{dia}}\right) /\left(\delta P / P_{\mathrm{tot}}\right) \approx 0.6$, in reasonable agreement with an estimated first pass absorption of second harmonic O-mode at the prevailing density and temperature of $45 \%$. Note that this efficiency is still higher that the expected efficiency of 0.3 taking into account the full injected ECRH power and the power degradation according to the RI mode scaling, equation (1).

With increasing density in the RI mode, the central sawtooth behaviour exhibits a marked change from normal to humpback like. This changeover to humpback like sawteeth in a standard RI mode discharge is shown in figure 20, which displays the central ECE in third harmonic X-mode (for these 


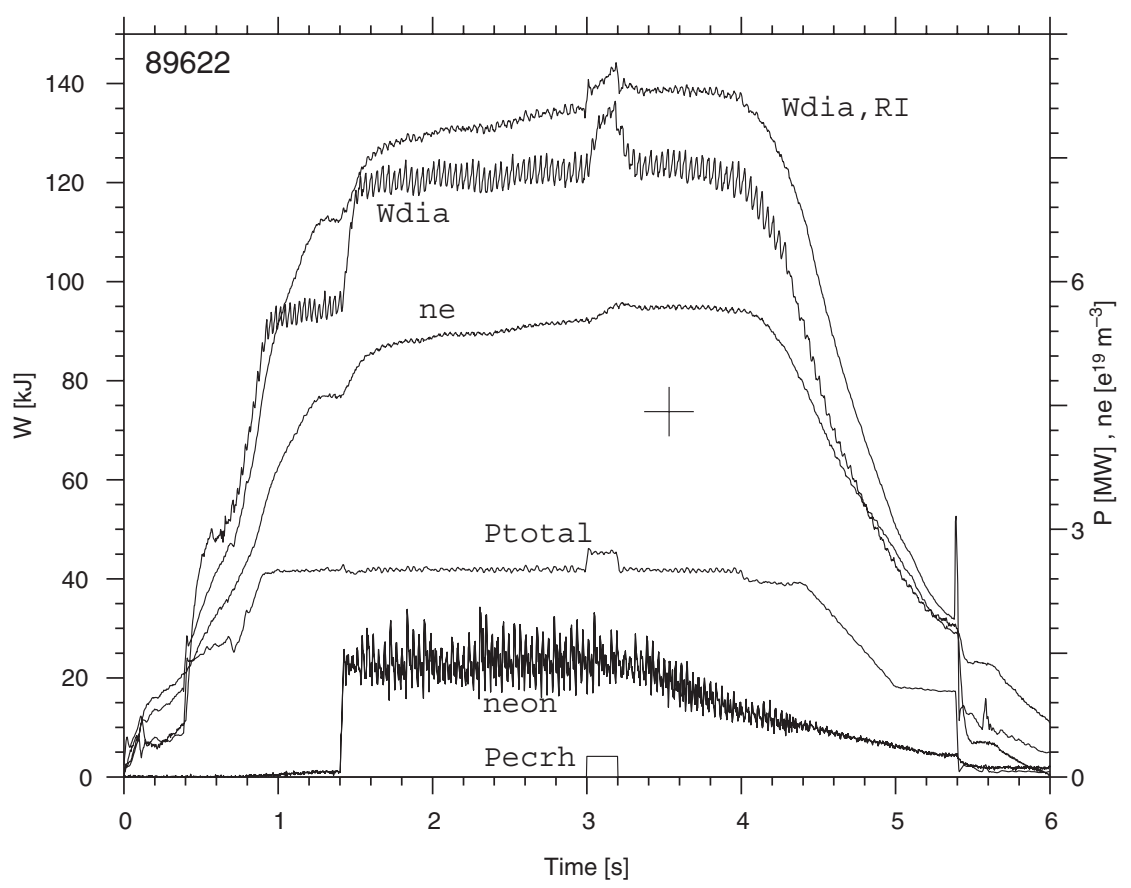

Figure 18. Evolution of a typical RI mode discharge. The increase of $W_{\text {dia }}$ with the injection of Ne signifies the transition to RI mode. The curve labelled $W_{\text {dia,RI }}$ signifies the expected $W$ from RI mode scaling [3].

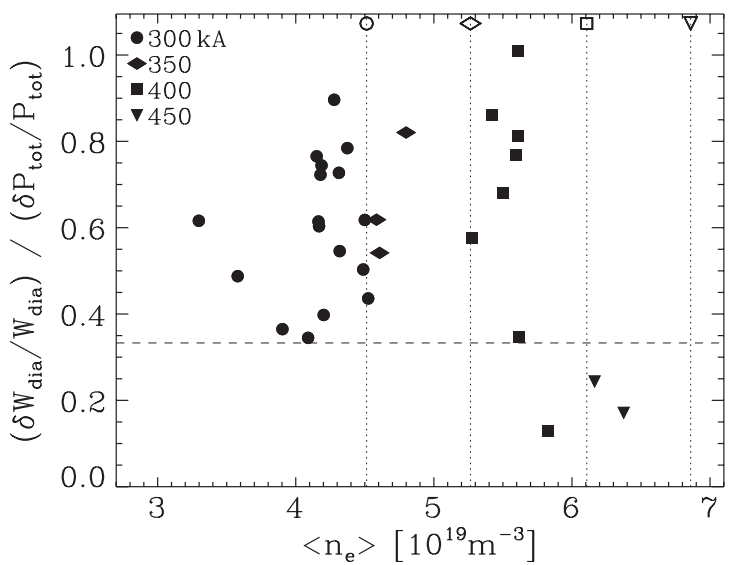

Figure 19. The normalized efficacy of ECRH in RI mode discharges. The different symbols indicate the plasma current. The dashed line is the efficacy expected by the RI mode scaling law. The vertical dashed lines indicate the Greenwald density for the four different values of the plasma current. The sharp drop of efficacy near $\bar{n}_{\mathrm{e}} \approx 5.5 \times 10^{19} \mathrm{~m}^{-3}$ is not caused by reaching the Greenwald density limit, but is due to the cut-off density.

higher densities the second harmonic ECE is affected by cut-off) together with the increasing line averaged density. Interestingly, with central ECRH the transition appears to be triggered at a slightly lower density. Figure 21 shows the central third harmonic ECE during ECRH in three different discharges: from bottom to top, a discharge at moderate density showing normal sawteeth with shorter period during central ECRH, a discharge close to the cut-off density showing first the short period normal sawteeth changing into humpback like towards the end of the ECRH pulse, and finally a discharge just above cut-off in which practically no effect of ECRH can be seen.

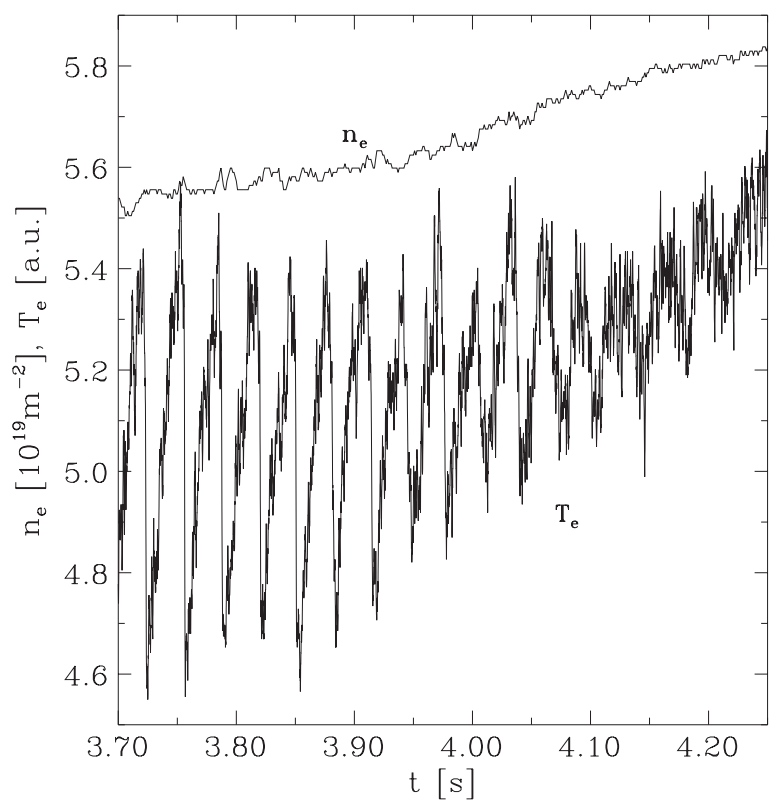

Figure 20. The sawtooth behaviour in a standard RI mode discharge measured with third harmonic X-mode ECE. With increasing density, the sawtooth behaviour changes from normal to humpback like. Note that the changeover occurs at a density just above the $110 \mathrm{GHz}$ X-mode cut-off.

In order to study local transport, scans of the normalized ECRH power deposition radius $\left(\rho_{\text {dep }}\right)$ were made by poloidal tilting of the ECRH launching mirror. Two scans were made one at $I_{\mathrm{p}}=405 \mathrm{kA}\left(q_{\mathrm{a}}=3.6\right)$ and $\bar{n}_{\mathrm{e}} \simeq 5.5 \times 10^{19} \mathrm{~m}^{-3}$, and the other at $I_{\mathrm{p}}=305 \mathrm{kA}\left(q_{\mathrm{a}}=4.8\right)$ and $\bar{n}_{\mathrm{e}} \simeq$ $4.2 \times 10^{19} \mathrm{~m}^{-3}$. Figure 22 shows the normalized efficacy of ECRH for the discharges of these two scans. If the electron thermal diffusivity $\left(\chi_{\mathrm{e}}\right)$ were a smooth function of minor 


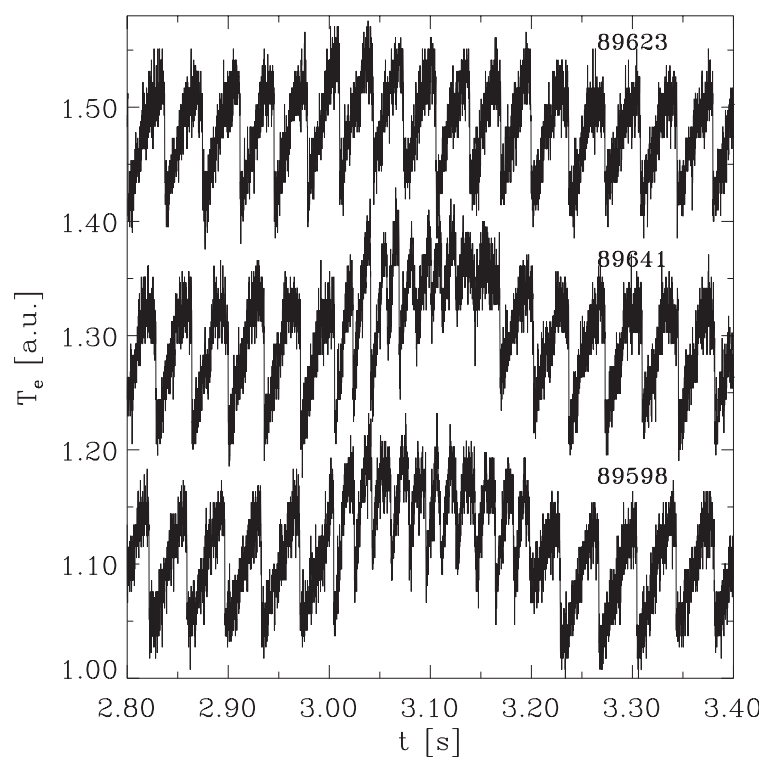

Figure 21. The sawtooth behaviour during central ECRH in RI mode at different densities. The density increases from well below cut-off, $\bar{n}_{\mathrm{e}}=4.6 \times 10^{19} \mathrm{~m}^{-3}$, for discharge 89598 (bottom trace) to above cut-off, $\bar{n}_{\mathrm{e}}=5.8 \times 10^{19} \mathrm{~m}^{-3}$, for 89623 (top trace). The middle trace, 89641 showing a transition to humpback like sawteeth during ECRH, is for a case close to cut-off, $\bar{n}_{\mathrm{e}}=5.6 \times 10^{19} \mathrm{~m}^{-3}$.

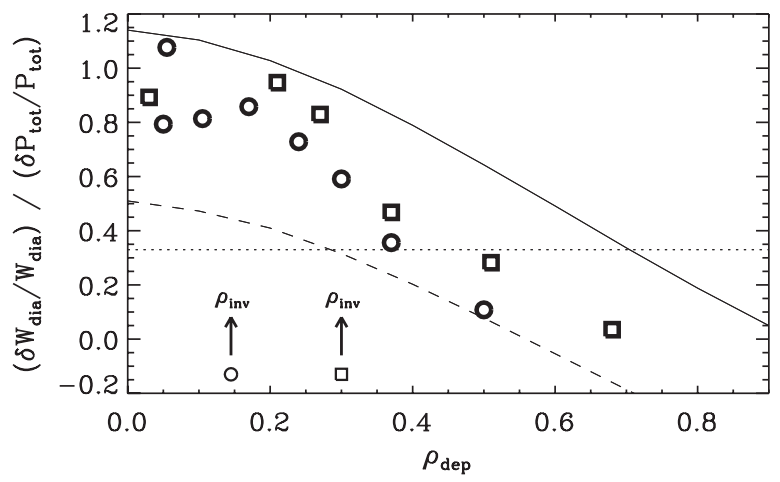

Figure 22. The normalized efficacy $\left(\delta W_{\mathrm{dia}} / W_{\mathrm{dia}}\right) /\left(\delta P / P_{\mathrm{tot}}\right)$ from diamagnetic measurements achieved in RI mode discharges as function of normalized ECRH deposition radius. The datasets shown are for $q_{\mathrm{a}}=3.8(\mathrm{O})$ and $q_{\mathrm{a}}=2.9(\square)$. The full (dashed) line indicates the expected efficacy of ECRH without (with) power degradation, assuming parabolic $\chi_{\mathrm{i}}=\chi_{\mathrm{e}}$ with $\chi_{\mathrm{e}}(0)=0.6$ and $\chi_{\mathrm{e}}(a)=3.2 \mathrm{~m}^{2} \mathrm{~s}^{-1}$. For both datasets a sharp drop of normalized efficacy is observed close to the sawtooth inversion radius (indicated by arrows), i.e. the footprint of a transport barrier in that region.

radius, a smooth decrease of the normalized efficacy of ECRH with increasing $\rho_{\text {dep }}$ would be observed. The full (dashed) line in figure 22 left shows the expected efficacy of ECRH without (with) power degradation, assuming a smooth $\chi_{\mathrm{e}}$ with a realistic radial profile. The narrow ECH power deposition, compared to NBI and ICRH, causes a calculated normalized efficacy greater than $1\left(\frac{1}{3}\right)$ for central ECRH without (with) power degradation. For both scans a high normalized efficacy, close to the expected value without power degradation, is observed up to $\rho_{\text {dep }} \sim 0.25$. Then the normalized efficacy drops sharply, indicating that there is a transport barrier near $\rho=0.3$. Neither a clear dependence of the barrier position on $q_{\mathrm{a}}$, nor a clear link between the sawtooth inversion radius

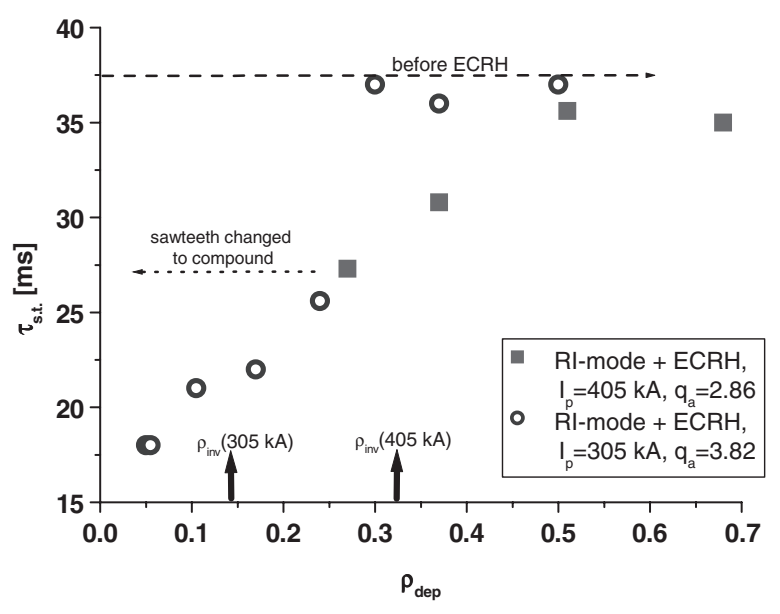

Figure 23. The sawtooth period as a function of the ECRH deposition radius $\rho_{\text {dep }}$ for the same data set as in figure 22. The sawtooth period is clearly reduced for heating inside the transport barrier. In the higher current case $I_{\mathrm{p}}=405 \mathrm{kA}$, sawteeth become compound during central ECRH when $\rho_{\text {dep }}<0.25$.

and the barrier is seen in RI mode. The sawtooth period is significantly reduced for heating inside the barrier, whereas the period is almost unaffected otherwise (see figure 23). In the higher current case, central ECRH leads to a change in the character of the sawteeth from normal to compound (with irregular partial and full collapses [30,31]).

\section{Conclusions and outlook}

Extensive studies covering a broad range of tokamak physics have been performed with the preliminary $110 \mathrm{GHz}, 270 \mathrm{~kW}$ (injected power), $200 \mathrm{~ms}$ gyrotron on TEXTOR. The most important conclusions are summarized as follows. Central ECRH on Ohmic target plasmas confirm the presence of an internal electron transport barrier close to the $q=1$ surface as seen previously on RTP [5]. This $q=1$ barrier is further corroborated by modulated ECRH $(\mathrm{MECH})$ experiments. Internal electron transport barriers are identified by ECRH during either the current ramp phase or in high confinement RI mode phase of TEXTOR discharges. In RI mode the internal transport barrier apparently prevents the expected degradation of confinement with the increasing power from ECRH: the relative change in diamagnetic energy is in proportion to the relative change in the total power. Thanks to the good focusing of the ECRH beam, central ECRH remains effective right up to the cut-off density for the second harmonic X-mode. Above $\mathrm{X}$-mode cut-off, surprisingly efficient heating with O-mode is found in spite of incomplete absorption. In the current ramp phase, central ECRH led to a faster current penetration and an early start of sawteeth near the end of ECRH, though only when combined with early heating from dominant counter-NBI. Either balanced or dominant co-NBI resulted in discharges with broader current density profiles, leading to $m=2, n=1$ modes finally resulting in a disruption. Control of sawteeth has been shown both for Ohmic as well as for RI mode target plasmas.

All these interesting results have been achieved with the old $110 \mathrm{GHz}$ gyrotron, which for the TEXTOR tokamak has rather limited power and pulse length. The installation of the 
new $140 \mathrm{GHz}, 800 \mathrm{~kW},>3 \mathrm{~s}$ gyrotron opens up a wealth of possibilities for further research in each of the experimental areas presented earlier.

Concerning central ECRH and internal electron transport barriers, the increased power will allow to search for transport barriers not only at $q=1$, but also at other low order rational surfaces. The ultimate goal for these investigations would be: (i) to verify or falsify the empirical finding on RTP that transport barriers occur at all low order rationals, and (ii) to manipulate the current density profile in order to optimize the enhanced confinement due to the barriers. The study of transport barriers in reversed or optimized shear regimes will also profit from the increased heating and current drive capabilities. For example, the higher power opens the possibility of achieving central shear reversal through counterECCD even during the current plateau phase of the discharge.

The studies of transport in RI-mode plasmas profit in several ways from the new system. The higher frequency and, consequently, higher cut-off density will allow effective heating also in the highest performance RI mode discharges. The new gyrotron will deliver a significant fraction of input power ( $\sim 30 \%$ instead of $\sim 10 \%$ ); it will be interesting to see whether the increase of stored energy still scales linearly with ECRH power. The higher power allows to perform on- and off-axis power modulation studies also in RI mode.

Finally, the longer pulse length and improved heating and current drive capabilities open wider possibilities for control of instabilities as sawteeth and (neoclassical) tearing modes. In this context, the magnetic islands that will be generated by the DED [1] form the perfect 'laboratory' for (feedback) control of magnetic islands by heating and current drive. Localized ECRH inside the DED ergodic zone will also be a valuable tool for the diagnosis of ergodic fields.

\section{Acknowledgments}

This work, supported by the European Communities under the contract of Association between EURATOM/FOM, was carried out within the framework of the European Fusion Programme. The views and opinions expressed herein do not necessarily reflect those of the European Commission.

\section{References}

[1] Finken K.H. et al 1999 Nucl. Fusion 39637

[2] Barth C.J. et al 2001 Rev. Sci. Intrum. 721138

[3] Weynants R.R. et al 1999 Nucl. Fusion 391637

[4] Bindslev H. et al Proc. 10th Int. Symp. on Laser-Aided Plasma Diagnostics (Fukuoka, Japan, 2001) pp 97-108
[5] Lopes Cardozo N.J. et al 1997 Plasma Phys. Control. Fusion 39 B303

[6] Hogeweij G.M.D., Lopes Cardozo N.J., de Baar M.R. and Schilham A.M.R. 1998 Nucl. Fusion 381881

[7] Verhoeven A.G.A. et al 1995 Proc. 9th Joint Workshop on ECE an ECRH (Borrego Springs, California, USA, 23-26 January 1995) p 557

[8] Oomens A.A.M. et al 1996 Proc. 19th Symp. on Fusion Technology (Lisbon, Portugal, 16-20 September 1996) p 557

[9] Oosterbeek J.W. et al 2002 The ECW installation at the TEXTOR tokamak 22nd Symp. on Fusion Technology (Helsinki, Finland, 9-13 September 2002)

[10] Hoekzema J.A. et al 2002 The ECW system on TEXTOR 27th IEEE International Conf. on Infrared and Millimeter Waves (San Diego, California, USA, 22-26 September 2002)

[11] Krom J.G. et al 2002 Fusion Eng. Des. 60475

[12] Kaye S.M. 1997 Nucl. Fusion 371303

[13] Schüller F.C. et al 2000 First results of ECRH on TEXTOR: filaments, barriers, and RI-mode 27th EPS Conf. on Control. Fusion and Plasma Physics (Budapest, Hungary, 12-16 June 2000) http://epsppd.epfl.ch/Buda/pdf/d1_002.pdf

[14] Jaspers R. et al 2003 Electron transport barriers in TEXTOR plasmas 30th EPS Conf. on Control. Fusion and Plasma Physics (St. Petersburg, Russia, 7-11 July 2003) P2.133

[15] Sillen R.M.J. et al 1986 Nucl. Fusion 26303

[16] Pietrzyk Z.A. et al 1999 Nucl. Fusion 39587

[17] Mantica P. et al 2000 Phys. Rev. Lett. 854534

[18] Koide Y. et al 1994 Phys. Rev. Lett. 723662

[19] Joffrin E. et al 2002 Nucl. Fusion 42235

[20] Razumova K.A. et al 2000 Plasma Phys. Control. Fusion 42973

[21] Wolf R.C. 2003 Plasma Phys. Control. Fusion 45 R1

[22] Günter S. et al 2000 Phys. Rev. Lett. 843097

[23] Pietrzyk Z.A. et al 2001 Phys. Rev. Lett. 861530

[24] Sips A.C.C. 1998 Plasma Phys. Control. Fusion 40647

[25] Westerhof E. et al 2001 Electron cyclotron resonance heating in the current ramp-up phase of TEXTOR-94 28th EPS Conf. on Control. Fusion and Plasma Physics (Madeira, Portugal, 18-22 June 2001) vol 25A (ECA) (Geneva: European Physical Society) CD-Rom Paper P1.014, http://epsppd.epfl.ch/Madeira/html/pdf/P1.014.pdf

[26] Krämer-Flecken A. et al 2002 Heterodyne ECE diagnostic in the mode detection and disruption avoidance at TEXTOR Nucl. Fusion submitted

[27] Tokar M. et al 1999 Plasma Phys. Control. Fusion 41 B317

[28] Jackson G.L. et al 2002 Plasma Phys. Control. Fusion 44 1893

[29] Hogeweij G.M.D. et al 2001 Confinement and transport in EC heated RI-mode discharges in TEXTOR-94 28th EPS Conf. on Control. Fusion and Plasma Physics (Madeira, Portugal, 18-22 June 2001) vol 25A (ECA) (Geneva: European Physical Society) CD-Rom Paper P4.013, http://epsppd.epfl.ch/Madeira/html/pdf/P4.013.pdf

[30] Campbell D.J. et al 1986 Nucl. Fusion 261085

[31] Westerhof E. 1987 Comments Plasma Phys. Control. Fusion 1163 